\title{
Dedicated Radio Utilization for Spectrum Handoff and Efficiency in Cognitive Radio Networks
}

\author{
A. Ozan Bicen, Student Member, IEEE, Ecehan B. Pehlivanoglu, Student Member, IEEE, Sebastià Galmés, \\ Member, IEEE, and Ozgur B. Akan, Senior Member, IEEE
}

\begin{abstract}
To perform spectrum handoff, cognitive radio (CR) nodes communicating with each other need to exchange licensed user detection information, i.e., perform spectrum coordination, over a common control channel. The spectrum coordination can be fulfilled either via existing cognitive radio interface with time division or via a separate dedicated radio, i.e., a common control interface (CCI), continuously. CR nodes with CCI can instantly exchange licensed user detection information and cease frame transmission, while spectrum coordination can only be performed after the frame transmission period without CCI. Nevertheless, the impact of CCI incorporation into $C R$ nodes in terms of common performance metrics must be thoroughly assessed to evaluate the worthiness of additional radio cost. In this paper, an analytical framework is presented to assess the impact of CCI incorporation into $C R$ nodes for spectrum handoff. The developed framework enables analyzing potential benefits and disadvantages of employing CCI for spectrum handoff, in terms of achievable delay, energy consumption, spectrum utilization and event estimation performance. Extensive performance evaluations are presented to illustrate the impact of CCI utilization on efficiency of spectrum handoff. The network and communication regimes that would yield having CCI favorable are characterized in terms of spectrum conditions and CR parameters.
\end{abstract}

Index Terms-Cognitive radio, spectrum handoff, common control interface, frame transmission duration, spectrum efficiency, energy efficiency, cognitive radio sensor networks.

\section{INTRODUCTION}

D YNAMIC spectrum access (DSA) techniques for wireless communications have emerged to mitigate inefficient spectrum utilization in fixed spectrum assignment policies. Cognitive Radio (CR) is the fundamental progress towards realization of DSA in wireless communication [1], [2]. CR nodes can tune their communication parameters autonomously, adapting to the medium conditions and communicating over vacant frequency bands in the spectrum.

Apart from improved spectrum utilization and communication capacity promises, CR brings the burden of intermittent communication to wireless networks. Since spectrum access is paused during spectrum handoff, communication is interrupted with licensed user, i.e., primary user (PU), arrival. Even with

The work of A. O. Bicen, E. B. Pehlivanoglu, and O. B. Akan are supported by the Turkish Scientific and Technical Research Council under grant \#110E249.

The work of S. Galmés is supported by the Spanish Ministry of Science and Innovation under contract TIN2010-16345.

A. O. Bicen, E. B. Pehlivanoglu, and O. B. Akan are with the Nextgeneration and Wireless Communications Laboratory (NWCL), Department of Electrical and Electronics Engineering, Koc University, Istanbul, Turkey 34450 (e-mail: \{abicen, epehlivanoglu, akan\}@ku.edu.tr).

S. Galmés is with the Department of Mathematics and Computer Science, University of Balearic Islands, Spain (e-mail: sebastia.galmes@uib.es). abundant spectrum opportunities, Cognitive Radio Network (CRN) communication is still interrupted by spectrum sensing functionality for PU detection. In case of limited spectrum availability, spectrum access and successful communication take significantly longer time. In such scenarios, consecutive channel switching and spectrum sensing periods take place until spectrum handoff is successfully completed at a vacant channel, imposing severe communication delay and spectrum and energy inefficiencies. In that sense, analysis and understanding of the intermittent communications in CRN with its effects on communication delay and efficiency are essential for network design and devising of communication algorithms.

Spectrum coordination between communicating parties, i.e. distributing spectrum sensing results and negotiating for spectrum decision over a common medium, is a must in CRN [3], [4], [5]. Spectrum coordination can be performed over the existing CR interface [4], [6], [7], [8], or over a dedicated Common Control Interface (CCI), which can be integrated into CR nodes [5], [9], [10]. Existence of CCI can make a difference in the efficiency of CRNs, depending on the spectrum and communication conditions. Without CCI, nodes cannot perform spectrum coordination during transmissions and have to wait for current transmission to end for further spectrum coordination. However, with CCI, spectrum coordination can be performed simultaneously with transmissions, allowing transmissions to be ceased instantly by PU detection information. Dedicated interface for spectrum coordination increases energy consumption, but savings in energy consumption by simultaneous spectrum coordination may compensate it in certain conditions. Goodput definitely increases with CCI due to decreased delay, however its extent has to be evaluated. All these effects of CCI would have nontrivial implications on distributed sensing in Cognitive Radio Sensor Networks (CRSN). Hence, possible efficiency improvements provided by incorporation of CCI into CR nodes must be thoroughly assessed according to spectrum conditions and CR parameters; such as PU activity, spectrum sensing and frame transmission durations and their respective power consumption levels. Such assessments are useful to find out when CCI is worthy of its cost to meet performance requirements.

In this paper, we present an analytical framework to assess the communication and distributed sensing efficiency impacts of CCI utilization for spectrum handoff in CRN. The developed framework reveals achievable delay, spectrum and energy efficiency in CRN with and without CCI. Gain in distributed event sensing distortion via CCI utilization is also analyzed for CRSN. To this end, successful frame 
transmission (SFT) duration is formulated for a CR node pair and its closed-form expressions, with and without CCI, for any given channel sequence under heterogeneous PU activity, wireless error rate, spectrum sensing and frame transmission durations are derived. The developed framework can also be used with any propagation model. Furthermore, taking SFT durations as a reference point, the energy consumption and spectrum efficiency with and without CCI in CRN are analytically characterized. SFT duration analyses are also embedded into event estimation distortion analysis in CRSN. Finally, performance evaluations regarding CCI employment are presented. Numerical results illustrate achievable gains by CCI incorporation and reveal spectrum and network conditions and CR parameters that yield CCI favorable.

The contributions of our paper are three fold. First, the effect of using a dedicated radio for spectrum coordination on successful frame transmission duration is analytically characterized for the first time in the literature. Second, unlike other works, the spectrum handoff performance assessment with and without CCI is not limited to the delay perspective. Energy and spectrum efficiency and distributed sensing performance under spectrum handoff with and without CCI are studied analytically. Finally, the impact of CCI is thoroughly assessed by numerical results, and the network and spectrum conditions for which its gains become significant are revealed. All these contributions constitute valuable design guidelines on whether or not the inclusion of CCI is justifiable, given the conditions on the communication environment and network properties.

The remainder of the paper is organized as follows: In Section II, the related work on spectrum handoff performance in CRN is presented. Section III contains the analysis on the time elapsed until a frame is successfully transmitted with and without CCI. Sections IV, V and VI analytically characterize the impact of CCI on energy and spectrum efficiency and distributed sensing performance. The effects of CCI on network performance are assessed in Section VII by extensive numerical evaluations and paper is concluded in Section VIII.

\section{RELATED WORK}

Here, we describe the existing works in the literature on the spectrum handoff performance in CRN and point out our work's significance. In [11], authors examine delay of multiple spectrum handoffs on the data communication duration. A preemptive resume priority (PRP) M/G/1 queueing system is proposed to model multiple handoff delay. Extended data delivery duration due to interruptions by PU arrivals is modelled to assist the progress in designing admission control strategies for secondary networks according to specific latency requirements. Instead of using CCI for coordination, a frequency hopping-based spectrum handoff scheme is proposed in [12]. The authors study delay caused by spectrum handoff process in ad hoc CRN under homogeneous PU activity at each channel. A three dimensional discrete-time Markov chain is proposed to capture behaviour of spectrum handoffs and assess performance of CR users according to communication requirements. In [13], a PRP M/G/1 queueing network model is also proposed to reveal spectrum utilization of connectionoriented spectrum handoffs. Extended data delivery time is derived, and latency performance is analyzed. In [14], authors investigate prioritized secondary user traffic. They consider a general setting in which the PU transmissions can happen at any time instant. Centralized and distributed schemes are considered to manage the prioritized secondary user traffic that use different handoff mechanisms. The proposed DSA schemes are analyzed using a continuous time Markov chain. The mean handoff delay, for different priority secondary users is derived. In [15], the effects of interruptions due to multiple spectrum handoffs on connection-based channel usage is investigated based on a PRP M/G/1 queueing model. In [16], spectrum handoff process is analyzed. The average number of spectrum handoffs is derived to assess spectrum handoff performance.

In [11]-[13] and [15]-[16] the impact of PU traffic on the SU communication delay is assessed, without CCI consideration. In other works, a CCI or a common control channel is included for coordination and rendezvous [14], [17] or for sharing spectrum sensing results [18]. Our work differs from the literature by assessing the benefits of CCI on the spectrum handoff performance in terms of communication delay, and its consequent impact on network performance metrics for CRN and distributed sensing performance of CRSN. None of the existing works highlight the potentials of CCI for spectrum handoff, and our work fills this gap on the analysis of the cost-benefit tradeoffs of CCI inclusion in CRN and CRSN.

\section{SuCCESSFUl Frame TRANSMission DURATION} ANALYSIS

In this section, we formulate successful frame transmission (SFT) durations with and without CCI and derive their probability density functions (p.d.f.s). SFT durations are prolonged by PU arrivals. Without CCI, PU communication can only be detected after completion of current frame transmission; whereas with CCI, secondary users can be instantly informed about PU communication by neighbour nodes during frame transmission. We first present the system model, continue with formulation of the spectrum access (SA) delay, i.e. the duration until an SU node gets a spectrum opportunity. Then, SFT durations with and without CCI are analytically characterized.

\section{A. System Model}

In CRN, unlicensed users, i.e. SUs, coexist with licensed users, i.e. PUs. Each SU has a CR interface, which supports channel switching and spectrum sensing. SUs perform periodic spectrum sensing to detect PU activity. When an SU performs spectrum sensing, it cannot transmit data. Upon detection of PU communication, SUs perform spectrum handoff. During spectrum handoff, SU moves to the next channel in its predetermined circular channel list, then spectrum sensing is restarted at this channel. If PU communication is not detected at a channel throughout a whole sensing period, SU resumes its communication in this channel, otherwise spectrum handoff is performed consecutively until a vacant channel is found.

The traversed channel list during spectrum handoff may be provided by a centralized spectrum sharing mechanism to CRN nodes, or it may be constructed by means of cooperative spectrum sensing, as well as each node may be required to 
TABLE I

Illustration of Spectrum ACCess Delay for a ChanNel List COMPOSED OF 3 CHANNELS

\begin{tabular}{c|c|c}
$\begin{array}{c}\text { \# of Visited } \\
\text { Channels }\end{array}$ & $\begin{array}{c}\text { Cumulative Spectrum } \\
\text { Access Delay }\end{array}$ & Probability \\
\hline 1 & $\tau_{s}$ & $P_{+}^{(1)}$ \\
\hline 2 & $\tau_{\text {is }}^{(1)}+\tau_{s}$ & $P_{-}^{(1)} \cdot P_{+}^{(2)}$ \\
\hline 3 & $\tau_{\text {is }}^{(1)}+\tau_{\text {is }}^{(2)}+\tau_{s}$ & $P_{-}^{(1)} \cdot P_{-}^{(2)} \cdot P_{+}^{(3)}$ \\
\hline 5 & $\tau_{\text {is }}^{(1)}+\tau_{\text {is }}^{(2)}+\tau_{\text {is }}^{(3)}+\tau_{\text {is }}^{(1)}+\tau_{s}$ & $P_{-}^{(1)} \cdot P_{-}^{(2)} \cdot P_{-}^{(3)} \cdot P_{-}^{(1)} \cdot P_{+}^{(2)}$ \\
\hline$\ldots$ & $\cdots$ & $\ldots$
\end{tabular}

decide individually on which channel is to be sensed next [19]. Formation of the optimal channel list is beyond the scope of this paper, and the effect of channel list on our analysis is normalized using the same channel list for both spectrum handoff with and without CCI.

We assume that PU activity for each channel follow an i.i.d. ON/OFF random process [20], [21], [22], [23]. The channel is occupied by a PU during an ON period. The OFF periods stand for PU absence at the channel. Inter-arrival and interdeparture times of PU with an arrival rate of $\lambda_{c}$ and departure rate of $\beta_{c}$ on a specific channel $c$ are exponentially distributed with a mean of $\frac{1}{\lambda_{c}}$ and $\frac{1}{\beta_{c}}$, respectively.

\section{B. SFT Duration without CCI}

To investigate SFT duration without CCI, first we analytically model the delay experienced by an SU until it seizes a spectrum opportunity in a predetermined circular list of channels. This delay comprises the cumulative elapsed time on a number of consecutive channels due to repetitive spectrum handoff, until a full spectrum sensing period is completed at one of the channels without PU interruption. Required spectrum sensing duration for each channel varies based on the channel signal-to-noise ratio (SNR) and PU arrival $\lambda_{c}$ and departure $\beta_{c}$ rates [23]. To reflect this randomness, we assume that the required spectrum sensing intervals $\left(\tau_{s}^{(c)}\right)$ for different channels are outcomes of a uniform random variable. We drop channel index $c$ from spectrum sensing interval and take spectrum sensing intervals for every channel $c$ in channel list at different access instants as an independent and identically distributed (i.i.d.) random variable $\tau_{s}$, distributed as

$$
f_{\tau_{s}}(\tau)= \begin{cases}\frac{1}{\tau_{s}^{\max }-\tau_{s}^{\min }}, & \tau_{s}^{\min } \leq \tau \leq \tau_{s}^{\max } \\ 0, & \text { otherwise }\end{cases}
$$

where $\tau_{s}^{\max }, \tau_{s}^{\min }$ are maximum and minimum values of $\tau_{s}$.

To determine the p.d.f. of SA delay $\tau_{\mathrm{sa}}$, we define the random variable $\tau_{\text {is }}^{(c)}$ for the duration of an interrupted spectrum sensing period in channel $c$. If a PU transmission occurs before the completion of the sensing period $\tau_{s}$, SU stops spectrum sensing and performs channel switching and restarts spectrum sensing in the next channel. This operation is repeated until a full spectrum sensing period is completed at a channel without any PU arrival. At this point, we say that spectrum is seized at that channel, right after which frame transmission begins. In accordance with the PU model described in Section
III-A, the conditional cumulative distribution function (c.d.f.) of interrupted sensing duration $\tau_{\text {is }}^{(c)}$ is given by

$$
\begin{aligned}
F_{\tau_{\mathrm{is}}^{(c)}}\left(\tau \mid \tau_{s}\right) & =\frac{P\left(t \leq \tau \cap t \leq \tau_{s}\right)}{P\left(t \leq \tau_{s}\right)} \\
& =\frac{1-e^{-\lambda_{c} \tau}}{1-e^{-\lambda_{c} \tau_{s}}}, \quad 0<\tau \leq \tau_{s}
\end{aligned}
$$

where $\lambda_{c}$ is the PU arrival rate in channel $c$. Taking derivative of (2) with respect to $\tau$, the conditional p.d.f. of $\tau_{\text {is }}^{(c)}$ is

$$
f_{\tau_{\mathrm{is}}^{(c)}}\left(\tau \mid \tau_{s}\right)=\frac{\lambda_{c} e^{-\lambda_{c} \tau}}{1-e^{-\lambda_{c} \tau_{s}}}, \quad 0<\tau \leq \tau_{s}
$$

Then, by removing conditioning using the p.d.f. of $\tau_{s}$ given in (1), the p.d.f. of $\tau_{\text {is }}^{(c)}$ is obtained for $0<\tau \leq \tau_{s}^{\min }$ as

$$
\begin{aligned}
f_{\tau_{\mathrm{is}}^{(c)}}(\tau) & =\int_{\tau_{s}^{\min }}^{\tau_{s}^{\max }} \frac{\lambda_{c} e^{-\lambda_{c} \tau}}{1-e^{-\lambda_{c} \tau_{s}}} \frac{1}{\tau_{s}^{\max }-\tau_{s}^{\min }} \mathrm{d} \tau_{s} \\
& =\frac{e^{-\lambda_{c} \tau}}{\tau_{s}^{\max }-\tau_{s}^{\min }} \ln \left(\frac{e^{\lambda_{c} \tau_{s}^{\max }}-1}{e^{\lambda_{c} \tau_{s}^{\min }}-1}\right)
\end{aligned}
$$

and for $\tau_{s}^{\min } \leq \tau \leq \tau_{s}^{\max }$ as

$$
\begin{aligned}
f_{\tau_{\mathrm{is}}^{(c)}}(\tau) & =\int_{\tau}^{\tau_{s}^{\max }} \frac{\left(\lambda_{c} e^{-\lambda_{c} \tau}\right)}{\left(1-e^{-\lambda_{c} \tau_{s}}\right)\left(\tau_{s}^{\max }-\tau_{s}^{\min }\right)} \mathrm{d} \tau_{s} \\
& =\frac{e^{-\lambda_{c} \tau}}{\tau_{s}^{\max }-\tau_{s}^{\min }} \ln \left(\frac{e^{\lambda_{c} \tau_{s}^{\max }}-1}{e^{\lambda_{c} \tau}-1}\right)
\end{aligned}
$$

Finally, $f_{\tau_{\text {is }}^{(c)}}$ can be expressed by

$$
f_{\tau_{\mathrm{is}}^{(c)}}(\tau)= \begin{cases}\frac{e^{-\lambda_{c} \tau}}{\tau_{s}^{\max }-\tau_{s}^{\min }} \ln \left(\frac{e^{\lambda_{c} \tau_{s}^{\max }}-1}{e^{\lambda_{c} \tau_{s}^{\min }}-1}\right), & 0 \leq \tau \leq \tau_{s}^{\min } \\ \frac{e^{-\lambda_{c} \tau}}{\tau_{s}^{\max }-\tau_{s}^{\min }} \ln \left(\frac{e^{\lambda_{c} \tau_{s}^{m a x}}-1}{e^{\lambda_{c} \tau}-1}\right), & \tau_{s}^{\min }<\tau \leq \tau_{s}^{\max } \\ 0, & \text { otherwise }\end{cases}
$$

Table I illustrates the SA delay $\tau_{\text {sa }}$ for different spectrum handoff cases, where $P_{+}^{(c)}$ and $P_{-}^{(c)}$ represent the probabilities of finding channel $c$ vacant and occupied, respectively. Channel switching delay $\tau_{\text {sw }}$ due to software-based hardware reconfiguration is counted in spectrum sensing duration $\tau_{s}$. Accordingly, $P_{+}^{(c)}$ during a spectrum sensing interval $\tau_{s}$ is

$$
\begin{aligned}
P_{+}^{(c)} & =\int_{\tau_{s}^{\min }}^{\tau_{s}^{\max }} e^{-\lambda_{c} \tau_{s}} \frac{1}{\tau_{s}^{\max }-\tau_{s}^{\min }} \mathrm{d} \tau_{s} \\
& =\frac{e^{-\lambda_{c} \tau_{s}^{\min }}-e^{-\lambda_{c} \tau_{s}^{\max }}}{\lambda_{c}\left(\tau_{s}^{\max }-\tau_{s}^{\min }\right)}
\end{aligned}
$$

Clearly, $P_{-}^{(c)}$ can be obtained by $1-P_{+}^{(c)} . \tau_{s}$ and $\tau_{\text {is }}^{(c)}$ are mutually independent random variables.

SA delay $\tau_{\mathrm{sa}}$ is shown to be the sum of independent $\tau_{\text {is }}$ random variables in Table I, and its p.d.f. is found by the convolution of the p.d.f.s of summed random variables as

$$
\begin{aligned}
f_{\tau_{\mathrm{sa}}}(\tau)= & {\left[1+\left(\prod_{c=1}^{C} P_{-}^{(c)}\right) f_{\tau_{\mathrm{sa}}^{\operatorname{tr}(C)}}(\tau)\right.} \\
& \left.+\left(\prod_{c=1}^{C} P_{-}^{(c)}\right)^{2}\left(f_{\tau_{\mathrm{sa}}^{\operatorname{tr}(C)}} * f_{\tau_{\mathrm{sa}}^{\operatorname{tr}(C)}}\right)(\tau)+\cdots\right] \\
& \cdot\left[\sum_{c=1}^{C}\left(\prod_{l=1}^{c-1} P_{-}^{(l)}\right) P_{+}^{(c)}\left(f_{\tau_{\mathrm{sa}}^{\operatorname{tr}(c-1)}} * f_{\tau_{s}}\right)(\tau)\right]
\end{aligned}
$$




\begin{tabular}{|c|c|c|c|c|c|}
\hline$\tau_{\text {icyc }}^{(I)}$ & & & $\tau_{i c y c}^{(3)}$ & \multicolumn{2}{|c|}{$\tau_{c y c}^{(l)}$} \\
\hline$\tau_{i s}^{(I)} \mid \tau_{s}$ & $\tau_{s}^{(2)}$ & $\tau_{i}$ & $\tau_{i s}^{(3)}$ & $\tau_{s}^{(l)}$ & $\tau_{f}$ \\
\hline PU arr & & & & & \\
\hline
\end{tabular}

Fig. 1. Illustration of SFT duration $\tau_{\mathrm{sft}}$.

where $\left(f_{\tau_{\mathrm{sa}}^{\mathrm{tr}(\mathrm{c}-1)}} * f_{\tau_{s}}\right)(\tau)$ is the conditional p.d.f. of SA delay given that channel $c$ is vacant, $f_{\tau_{\mathrm{st}}^{\operatorname{tr}(\mathrm{c}-1)}}(\tau)$ is the p.d.f. of the total duration spent for seeking a vacant channel until spectrum sensing starts at channel $c$ and is equal to $f_{\tau_{\mathrm{is}}^{(1)}} * f_{\tau_{\mathrm{is}}^{(2)}} * \cdots * f_{\tau_{\mathrm{is}}^{(c-1)}}$. For ease of analysis, convolutions can be turned into multiplication by taking the Laplace transform of the expression. In the resulting Laplace domain expression, since $\left|\prod_{c=1}^{C}\left(P_{-}^{(c)} f_{\tau_{\mathrm{is}}^{(\mathrm{c})}}(s)\right)\right|<1, \mathcal{F}_{\tau_{\mathrm{sa}}}(s)$ can be found via geometric series interpretation on infinite series as

$$
\mathcal{F}_{\tau_{\mathrm{sa}}}(s)=\frac{\sum_{c=1}^{C}\left(\prod_{l=1}^{c-1} P_{-}^{(l)}\right) P_{+}^{(c)}\left(\prod_{k=1}^{c-1} \mathcal{F}_{\tau_{\mathrm{is}}^{(\mathrm{k})}}(\tau)\right) \mathcal{F}_{\tau_{\mathrm{s}}}(s)}{1-\prod_{c=1}^{C}\left(P_{-}^{(c)} \mathcal{F}_{\tau_{\mathrm{is}}^{(\mathrm{c})}}(s)\right)},
$$

where $\mathcal{F}_{\tau_{\mathrm{sa}}}(s), \mathcal{F}_{\tau_{\mathrm{s}}}(s)$ and $\mathcal{F}_{\tau_{\mathrm{is}}^{(\mathrm{c})}}(s)$ are the Laplace transforms of $f_{\tau_{\mathrm{sa}}}(\tau), f_{\tau_{\mathrm{s}}}(\tau)$ and $f_{\tau_{\mathrm{is}}^{(\mathrm{c})}}(\tau)$, respectively. It is assumed that $P_{-}^{(0)}=1$ and $f_{\tau_{\text {is }}^{(0)}}(s)=1$.

To extend our SA delay analysis to SFT duration for the without CCI case, we define a new random variable $\tau_{\text {sft }}^{\mathrm{sa}}$, corresponding to having $k-1$ frame transmission trials, each interrupted by PU arrivals during transmission periods, before having a successful frame transmission. Then, the mean of the SFT duration without CCI, i.e. $\mathrm{E}\left\{\tau_{\mathrm{sft}}^{\mathrm{sa}}\right\}$, is given by

$$
\mathrm{E}\left\{\tau_{\mathrm{sft}}^{\mathrm{sa}}\right\}=\sum_{k=1}^{\infty} k\left(\mathrm{E}\left\{\tau_{\mathrm{sa}}\right\}+\tau_{f}\right) e^{-\lambda_{k} \tau_{f}} \prod_{c=1}^{k-1}\left(1-e^{-\lambda_{c} \tau_{f}}\right)
$$

where $k$ is the number of frame transmissions until successfully delivering a single frame, and $\tau_{f}$ is the deterministic duration for transmission of a single frame.

\section{SFT Duration with CCI}

Here, we analytically model the SFT duration with CCI, i.e. $\tau_{\text {sft }}$, and derive its p.d.f.. This duration comprises the cumulative elapsed time, both for spectrum sensing and frame transmission, until a frame is successfully delivered on the link. We call the duration of $\tau_{s}^{(c)}+\tau_{f}$ as the cognitive cycle duration $\tau_{\text {cyc. }}^{(c)}$. Each cognitive cycle $\tau_{\text {cyc }}^{(c)}$ in channel $c$ of the pre-defined circular channel list starts with a spectrum sensing interval $\tau_{\mathrm{s}}^{(c)}$ to seek a spectrum opportunity, and if an opportunity is found, the frame transmission interval $\tau_{f}$ starts, otherwise spectrum handoff to the next channel takes place. If PU arrival does not occur during the frame transmission period, the frame is said to be successfully transmitted, otherwise it is discarded and retransmitted with next spectrum opportunity. Different from the without CCI case, frame transmission can also be ceased upon PU arrival, via spectrum coordination over dedicated radio, i.e., CCI.

Since $\tau_{\mathrm{s}}^{(c)}$ is an i.i.d. random variable and $\tau_{f}$ is deterministic, $\tau_{\text {cyc }}^{(c)}$ is also an i.i.d. random variable for each channel at each access instant, and hence, channel index $c$ is dropped from it. Using (1), p.d.f. of $\tau_{\text {cyc }}^{(c)}$ is found as

$$
f_{\tau_{\mathrm{cyc}}}(\tau)= \begin{cases}\frac{1}{\tau_{\mathrm{cyc}}^{\max }-\tau_{\mathrm{cyc}}^{\min }}, & \tau_{\mathrm{cyc}}^{\min } \leq \tau \leq \tau_{\mathrm{cyc}}^{\max } \\ 0, & \text { otherwise }\end{cases}
$$

where $\tau_{\text {cyc }}^{\max }$ and $\tau_{\text {cyc }}^{\min }$ are equal to $\tau_{s}^{\max }+\tau_{f}$, and $\tau_{s}^{\min }+\tau_{f}$.

To determine the distribution of $\tau_{\mathrm{sft}}$, we define the random variable $\tau_{\text {icyc }}^{(c)}$ for interrupted cognitive cycle duration in channel $c$. The probability of finding channel $c$ in an ON, i.e., busy, and OFF, i.e., hole, state are $P_{b}^{(c)}=\frac{\lambda_{c}}{\lambda_{c}+\beta_{c}}$, and $P_{h}^{(c)}=\frac{\beta_{c}}{\lambda_{c}+\beta_{c}}$, respectively. Similar to derivation of $\tau_{\text {is }}^{(c)}$ in Section III-B, $\tau_{\text {icyc }}$ is found to be distributed by

$$
f_{\tau_{\text {icyc }}^{(c)}}(\tau)= \begin{cases}\frac{e^{-\lambda_{c} \tau}}{\tau_{\text {cyc }}^{\max }-\tau_{\text {cyc }}^{\min }} \ln \left(\frac{e^{\lambda_{c} \tau_{\text {cyc }}^{\max }}-1}{e^{\lambda_{c} \tau_{c y c}^{\min }}-1}\right), & 0<\tau \leq \tau_{\mathrm{cyc}}^{\min } \\ \frac{e^{-\lambda_{c} \tau}}{\tau_{\mathrm{cyc}}^{\max }-\tau_{\text {cyc }}^{\min }} \ln \left(\frac{e^{\lambda_{c} \tau_{\mathrm{cyc}}^{\max }}-1}{e^{\lambda_{c} \tau}-1}\right), & \tau_{\mathrm{cyc}}^{\min } \leq \tau \leq \tau_{\mathrm{cyc}}^{\max } \\ 0, & \text { otherwise }\end{cases}
$$

Fig. 1 illustrates SFT duration with CCI $\tau_{\text {sft }}$, whose p.d.f. $f_{\tau_{\text {sft }}}(\tau)$ is found as

$$
\begin{aligned}
f_{\tau_{\mathrm{sft}}}(\tau)= & {\left[1+\left(\prod_{c=1}^{C} P_{b}^{(c)}\right) f_{\tau_{\mathrm{st}}^{\operatorname{tr}(\mathrm{C})}}(\tau)\right.} \\
& +\left(\prod_{c=1}^{C} P_{\mathrm{b}}^{(c)}\right)^{2}\left(f_{\tau_{\mathrm{sft}}^{\operatorname{tr}(\mathrm{C})}} * f_{\left.\tau_{\mathrm{sft}}^{\operatorname{tr}(\mathrm{C})}\right)}(\tau)+\ldots\right] \\
& \cdot\left[\sum_{c=1}^{C}\left(\prod_{l=1}^{c-1} P_{b}^{(l)}\right) P_{h}^{(c)}\left(f_{\tau_{\mathrm{sft}}^{\operatorname{tr}(\mathrm{c}-1)}} * f_{\tau_{c y c}}\right)(\tau)\right]
\end{aligned}
$$

where $\left(f_{\tau_{\mathrm{sft}}^{\mathrm{tr}(c-1)}} * f_{\tau_{c y c}}\right)(\tau)$ is the conditional p.d.f. of SFT duration given that $c^{\text {th }}$ accessed channel is a spectrum hole. $f_{\tau_{\mathrm{sft}}^{\operatorname{tr}(c-1)}}(\tau)$ is the p.d.f. of the total interrupted cognitive cycle durations until cognitive cycle starts at channel $c$, which equals to $f_{\tau_{\text {icyc }}^{(1)}} * f_{\tau_{\text {icyc }}^{(2)}} * \ldots * f_{\tau_{\text {icyc }}^{(c-1)}}$. Following the derivation of $\mathcal{F}_{\tau_{\mathrm{sa}}}(s)$ in Section III-B, since $\left|\prod_{c=1}^{C}\left(P_{b}^{(c)} \mathcal{F}_{\tau_{\text {icyc }}^{(c)}}(s)\right)\right|<1$, $\mathcal{F}_{\tau_{\text {sft }}}(s)$ can be found via Laplace transform of (13) as

$$
\mathcal{F}_{\tau_{\mathrm{sft}}}(s)=\frac{\sum_{c=1}^{C}\left(\prod_{l=1}^{c-1} P_{b}^{(l)}\right) P_{h}^{(c)}\left(\prod_{k=1}^{c-1} \mathcal{F}_{\tau_{\text {icyc }}^{(k)}}(s)\right) \mathcal{F}_{\tau_{\text {cyc }}}(s)}{1-\prod_{c=1}^{C}\left(P_{b}^{(c)} \mathcal{F}_{\tau_{\text {icyc }}^{(c)}}(s)\right)},
$$

where $\mathcal{F}_{\tau_{\text {sft }}}(s), \mathcal{F}_{\tau_{\text {cyc }}}(s)$, and $\mathcal{F}_{\tau_{\text {icyc }}^{(c)}}(s)$ are the Laplace transforms of $f_{\tau_{\text {sft }}}(\tau), f_{\tau_{\text {cyc }}}(\tau)$ and $f_{\tau_{\text {icyc }}^{(c)}}(\tau)$, respectively. It is assumed that $P_{b}^{(0)}=1$ and $f_{\tau_{\text {icyc }}^{(0)}}(s)=1$. 
Eq. (10) and (13) are used to assess gains in delay performance via incorporation of CCI into CRN nodes in Section VII, and are also the starting point for the formulation of energy and spectrum efficiency and distributed event sensing performance analyses in Sections IV, V and VI, respectively.

\section{ENERGY EFFICIENCY ANALYSIS}

While CCI saves energy consumption by instant cessation of data transmission in case of PU arrival, it consumes extra energy as a dedicated radio. To investigate whether CCI utilization brings in energy efficiency, we study SFT energy consumption with and without CCI. Power consumption levels for spectrum sensing, main radio frame transmission and CCI radio operation are denoted as $\Upsilon_{s}, \Upsilon_{f}$ and $\Upsilon_{c c i}$ respectively. The mean energy consumption for an SFT without CCI is then

$\Omega_{\mathrm{sft}}^{\mathrm{sa}}=\sum_{k=1}^{\infty} k\left(\mathrm{E}\left\{\tau_{\mathrm{sa}}\right\} \cdot \Upsilon_{s}+\tau_{f} \cdot \Upsilon_{f}\right) \cdot e^{-\lambda_{c} \tau_{f}}\left(1-e^{-\lambda_{c} \tau_{f}}\right)^{k-1}$

Energy efficiency of SFT without CCI, i.e. the ratio of useful to total energy consumption is defined as $\Gamma_{\mathrm{sft}}^{\mathrm{sa}}=\left(\Upsilon_{f} \tau_{f}\right) / \Omega_{\mathrm{sft}}^{\mathrm{sa}}$.

On the other hand, with CCI, assuming CCI is kept off during spectrum sensing and is always on during transmissions, we can approximate the total energy consumption as

$$
\Omega_{\mathrm{sft}}=\mathrm{E}\left\{\tau_{\mathrm{ss}}\right\} \cdot \Upsilon_{s}+\left(\mathrm{E}\left\{\tau_{\mathrm{sft}}\right\}-\mathrm{E}\left\{\tau_{\mathrm{ss}}\right\}\right) \cdot\left(\Upsilon_{f}+\Upsilon_{c c i}\right)
$$

where $\mathrm{E}\left\{\tau_{\mathrm{ss}}\right\}=\mathrm{E}\left\{\tau_{\mathrm{sa}}\right\} \cdot \frac{\mathrm{E}\left\{\tau_{\mathrm{stt}}^{\mathrm{sa}}\right\}}{\mathrm{E}\left\{\tau_{\mathrm{sa}}+\tau_{f}\right.}$ is the total time spent on spectrum sensing until successfully transmitting a frame. Energy efficiency with CCI is then $\Gamma_{\mathrm{sft}}=\left(\Upsilon_{f} \tau_{f}\right) / \Omega_{\mathrm{sft}}$. Using $\Gamma_{\mathrm{sft}}$ and $\Gamma_{\mathrm{sft}}^{\mathrm{sa}}$, we elaborate and compare energy efficiency figures with and without CCI in Section VII-C.

\section{Spectrum EfFiciency Analysis}

Here, we formulate the spectrum utilization with and without CCI. Assuming that SU always has data to send, transmission starting at $t=0$ successfully delivers $D(t)$ frames and achieves a goodput of $G(t)=D(t) \cdot R \cdot \tau_{f} / t$ in a duration of $t$ for a communication rate of $R$. Steady-state goodput $G$ of such spectrum utilization is found as

$$
G=\lim _{t \rightarrow \infty} \frac{\mathrm{E}\{D(t)\} \cdot R \cdot \tau_{f}}{t}
$$

where $\mathrm{E}\{D(t)\}$ is the mean of the number of frames transmitted successfully in $[0, t]$ for $t \rightarrow \infty$.

Achieved goodput for an SFT, i.e., $D(t)=1$, in the duration of $\tau_{\mathrm{sft}}^{\mathrm{sa}}(i)$, i.e., in a realization of i.i.d random variable for SFT duration without CCI, is $G_{\mathrm{sft}}^{\mathrm{sa}}=R \cdot\left(\tau_{f} / \tau_{\mathrm{sft}}^{\mathrm{sa}}(i)\right)$. Since each $\tau_{\mathrm{sft}}^{\mathrm{sa}}(i)$ ends with an SFT, the evolution of SFT duration $\tau_{\mathrm{sft}}^{\mathrm{sa}}(i)$ can be taken as a Markov regenerative process [24] with a reward of SFT. From the renewal theorem, asymptotic goodput without CCI is $G_{\mathrm{sft}}^{\mathrm{sa}}=R \cdot\left(\tau_{f} / \mathrm{E}\left\{\tau_{\mathrm{sft}}^{\mathrm{sa}}\right\}\right)$ where $\mathrm{E}\left\{\tau_{\mathrm{sft}}^{\mathrm{sa}}\right\}$ is the mean of SFT duration without CCI derived in Section III-B. Similarly, asymptotic goodput with CCI is $G_{\mathrm{sft}}=R$. $\left(\tau_{f} / \mathrm{E}\left\{\tau_{\mathrm{sft}}\right\}\right)$ where $\mathrm{E}\left\{\tau_{\mathrm{sft}}\right\}$ is the mean of SFT duration with CCI derived in Section III-C. We use $G_{\mathrm{sft}}^{\mathrm{sa}}$ and $G_{\mathrm{sft}}$ to study role of CCI on spectrum efficiency in Section VII-D.

\section{Distributed Event Sensing Distortion ANALYSIS}

CRSNs may utilize the salient features of DSA and provide distributed sensing over licensed bands [25], [26], [27], [28], [29], [30], [31]. CRSN nodes report their event observations to the sink when they find spectrum opportunities. Using opportunistically collected samples from sensors, sink generates an estimate of the sensed signal, i.e., event, at the end of each estimation interval. Here, we assess the distributed sensing performance with and without CCI utilization in CRSN.

The mean event communication duration in an estimation interval $\tau_{e}$ is $\mathrm{E}\left\{\tau_{e}^{\mathrm{non}}\right\}=\left\lfloor\tau_{e} / \mathrm{E}\left\{\tau_{\mathrm{sft}}^{\mathrm{sa}}\right\}\right\rfloor \tau_{f}$ and $\mathrm{E}\left\{\tau_{e}^{\text {cci }}\right\}=$ $\left\lfloor\tau_{e} / \mathrm{E}\left\{\tau_{\mathrm{sft}}\right\}\right\rfloor \tau_{f}$ for without and with CCI cases, respectively. This duration reveals the average time spent for communication of event features with fusion center. We use $\mathrm{E}\left\{\tau_{e}^{\mathrm{cci}}\right\}$ and $\mathrm{E}\left\{\tau_{e}^{\text {non }}\right\}$ to calculate received samples from sensor nodes at sink at the end of each estimation interval.

We formulate estimation distortion with respect to event communication duration in an estimation interval under opportunistic spectrum access in CRSN. For estimation distortion analysis, the best linear unbiased estimator (BLUE) [32] is used to assess distortion without any specific assumption on the distribution of sensed signal and sensing noise. Sensor $k$ obtains distorted version $s_{k}(t)$ of the event signal $\theta(t)$ at time $t$ due to signal attenuation factor $h_{k}(t)$ and sensing noise $n_{s}^{k}(t)$, i.e., $s_{k}(t)=h_{k}(t) \theta(t)+n_{k}^{s}(t)$. We also assume that $h_{k}(t)$, $\theta(t)$ and $n_{k}^{s}(t)$ are i.i.d. over time. $\hat{\theta}(t)$ is estimated from the received samples from sensor nodes. We also assume that the mean of $\theta(t)$ and $n_{k}^{s}(t)$ are zero, and sensing noise $n_{k}^{s}(t)$ has a power of $\sigma_{k}^{2}$. Time indices are dropped in accordance with independence over time assumption. Using formulated event communication duration, number of received samples $X$ from sensor $k$ is found as $X_{\text {non }}=\left(\mathrm{E}\left\{\tau_{e}^{\text {non }}\right\} / \tau_{f}\right) \cdot S_{f}$ and $X_{\mathrm{cci}}=\left(\mathrm{E}\left\{\tau_{e}^{\mathrm{cci}}\right\} / \tau_{f}\right) \cdot S_{f}$ for without and with CCI cases, where $S_{f}$ is the number of samples per frame. Received sample vector $\mathbf{r}_{k}$ from sensor $k$ is as $\mathbf{r}_{\mathbf{k}}=\mathbf{h}_{\mathbf{k}} \theta+\mathbf{n}_{\mathbf{k}}^{\mathbf{s}}$, where $\mathbf{r}_{\mathbf{k}}=\left[r_{1}, \cdots, r_{X_{k}}\right]^{\mathrm{T}}, \mathbf{h}_{\mathbf{k}}=\left[h_{1}, \cdots, h_{X_{k}}\right]^{\mathrm{T}}$, and $\mathbf{n}_{\mathbf{k}}^{\mathbf{s}}=\left[n_{1}^{s}, \cdots, n_{X_{k}}^{s}\right]^{\mathrm{T}}$. Covariance matrix $\mathbf{R}_{\mathbf{k}}$ for received samples from CRSN node $k$ is a $X_{k}$ dimensional rectangular diagonal matrix whose diagonal entries are $\sigma_{k}^{2}$ for $1 \cdots X_{k}$, and other entries are zero. Hence, the mean square error (MSE) for sensor $k$ can be determined as [32]

$$
\begin{aligned}
\operatorname{Var}\left[\hat{\theta}_{k}\right] & =\left[\mathbf{h}_{\mathbf{k}}^{\mathrm{T}} \mathbf{R}_{\mathbf{k}}{ }^{-1} \mathbf{h}_{\mathbf{k}}\right]^{-1} \\
& =\left(\sum_{i=1}^{X_{k}} \frac{1}{\sigma_{k}^{2}}\right)^{-1}
\end{aligned}
$$

and overall estimation distortion at sink for the BLUE is

$$
\operatorname{Var}[\hat{\theta}]=\left(\sum_{k=1}^{K} \frac{X_{k}}{\sigma_{k}^{2}}\right)^{-1}
$$

We use (19) for distortion assessment of distributed sensing over CRSN with and without CCI in Section VII-E.

\section{ViI. Performance Evaluation}

In this section, CCI utilization for spectrum handoff is investigated numerically from SFT duration, energy efficiency, 


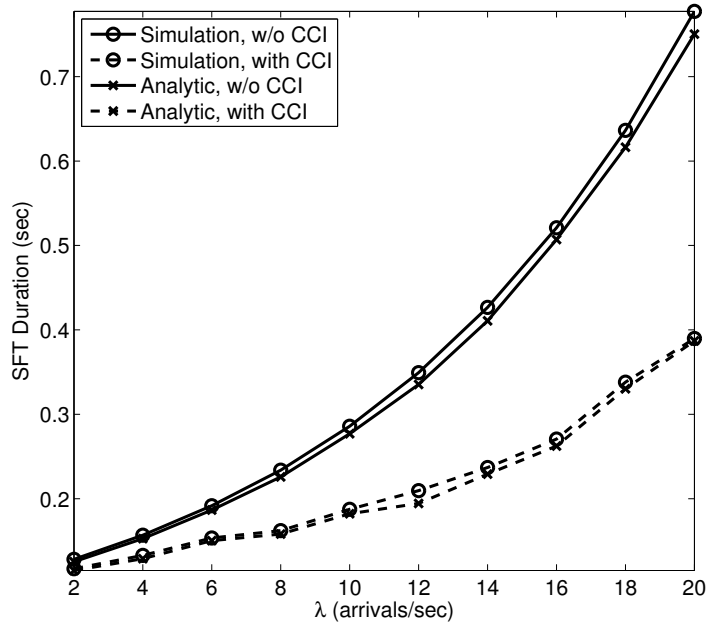

Fig. 2. Validation of SFT duration with and without CCI via time-step simulation.

spectrum efficiency and event estimation performance perspectives. We first give our network setup and investigate accuracy of the presented delay analysis in Section III. Then, effects of CCI utilization on spectrum handoff efficiency are examined.

\section{A. Network Setup}

For the assessment, frame duration $\tau_{f}$ and mean spectrum sensing duration $\mathrm{E}\left\{\tau_{s}\right\}$ are assumed to be $100 \mathrm{~ms}$ and 5 ms [36], PU packet arrival rate $\lambda$ is varied from 2 to 20 packets/sec, SNR for AWGN channels is assumed to be 20 $\mathrm{dB}$, unless otherwise stated.

1) The Nearest-neighbor Model: Nodes are assumed to communicate to their nearest neighbor. We assume that both PU and SU nodes are distributed randomly according to a stationary homogeneous Poisson Point Process (PPP), with intensities $\lambda_{\mathrm{PU}}$ and $\lambda_{\mathrm{SU}}$, respectively. With PPP, probability that an SU node has less than $\mathrm{k}$ neighbours in a neighbourhood of $r$ is $P(k)=\sum_{i=0}^{k-1} e^{-\lambda_{\mathrm{SU}} \pi r^{2}} \frac{\left(\lambda_{\mathrm{SU}} \pi r^{2}\right)^{i}}{i !}$. The p.d.f. for the distance to the nearest $\mathrm{SU}$ neighbour is then $f_{r_{1}}=$ $2 \pi \lambda_{\mathrm{SU}} r_{1} \cdot e^{-\lambda_{\mathrm{SU}} \pi r_{1}{ }^{2}}$ [33]. Given the distance $r_{1}$ between two neighbor nodes, and assuming each PU node has an average packet arrival rate of $\alpha$ arrivals/second, we approximate the total PU traffic in channel $c$ by arrivals in a radius $R_{c}=2 r_{1}$ neighborhood of the SU link as $\mathrm{E}\left\{\lambda_{R_{c}}\right\}=\alpha\left(\lambda_{\mathrm{PU}} \pi R_{c}^{2}\right)$.

2) Wireless Channel Model: Effects of CCI are studied for both AWGN and flat fading channels. Quadrature Amplitude Modulation (4-QAM) is assumed for SU transmissions. Bit error rate (BER) expression for AWGN channel under 4-QAM is $P_{b}^{\text {awgn }}=\frac{3}{4} Q\left(\sqrt{4 \frac{E_{b}}{N_{0}}} / 5\right)$, where $E_{b}$ is energy per bit and noise has flat PSD $N_{0} / 2$ [34]. The BER for 4-QAM over a fading channel $\left(P_{b}^{\text {fade }}\right)$ is given by $P_{b}^{\text {fade }}=\frac{2}{\pi} \int_{0}^{\pi / 2} M_{\gamma}\left(-\frac{9}{2 \sin ^{2}(\phi)}\right) \mathrm{d} \phi-$ $\frac{1}{\pi} \int_{0}^{\pi / 4} M_{\gamma}\left(-\frac{9}{2 \sin ^{2}(\phi)}\right) \mathrm{d} \phi$, where $M_{\gamma}(s)=(1-s \bar{\gamma})^{-1}$ and $\bar{\gamma}$ is the SNR [35].

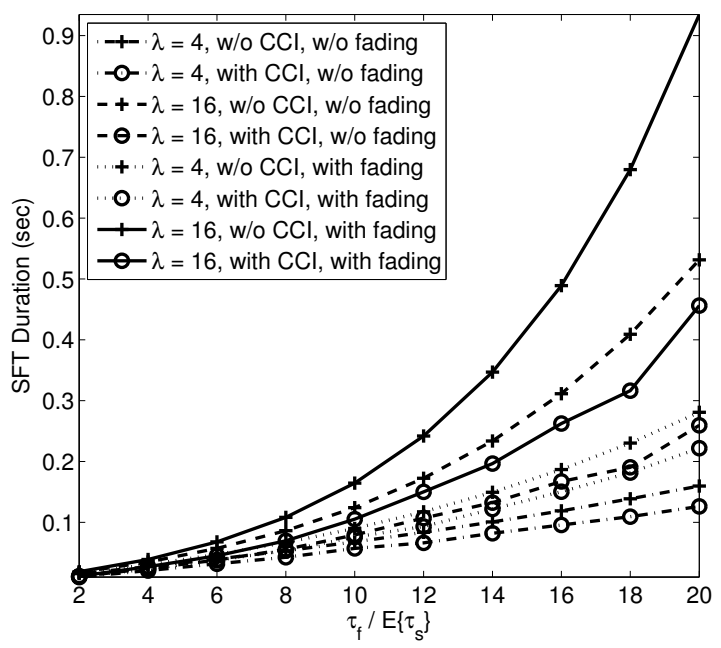

Fig. 3. SFT duration with and w/o CCI, for different PU arrival rates and $\frac{\tau_{f}}{\mathrm{E}\left\{\tau_{s}\right\}}$ ratios.

\section{B. SFT Duration}

Accuracy of the analytical expressions for $\mathrm{E}\left\{\tau_{\mathrm{sft}}^{\mathrm{sa}}\right\}$ and $\mathrm{E}\left\{\tau_{\mathrm{sft}}\right\}$, derived in Section III are first evaluated via timestep simulations. Then, we investigate and compare the SFT durations under the implications of the network model.

1) Validation of Delay Analyses: For validation, the analytical expressions are constructed in Mathematica and their results are compared with results of a realistic time-step simulation framework in MATLAB. The PU packet departure rate is set to 30 , in conformity with notion of stable channel. The analytical and simulation results for $\mathrm{E}\left\{\tau_{\mathrm{sft}}^{\mathrm{sa}}\right\}$ and $\mathrm{E}\left\{\tau_{\mathrm{sft}}\right\}$ are given in Fig. 2. The error between the analytical and simulation result values are below $5 \%$ for all given $\lambda$ values, increasing slightly as $\lambda$ 's become comparable to the $\beta$ value.

2) SFT Duration with and without CCI: We first define the SFT durations under the network setup, which are denoted by $\tau_{\text {cci }}$ and $\tau_{\mathrm{n}-\mathrm{cci}}$ for the cases with and without CCI, respectively. Assumption here is that whole $\tau_{\mathrm{sft}}$ and $\tau_{\mathrm{sft}}^{\mathrm{sa}}$ durations have to be repeated in case of a wireless error in these two scenarios. $\mathrm{E}\left\{\tau_{\text {cci }}\right\}$ is given by $\mathrm{E}\left\{\tau_{\text {cci }}\right\}=\sum_{k=1}^{\infty} k \cdot \mathrm{E}\left\{\tau_{\mathrm{sft}}\right\}$. $P_{\mathrm{cf}}\left(1-P_{\mathrm{cf}}\right)^{k-1}$ where $P_{\mathrm{cf}}=(1-\mathrm{BER})^{R \cdot \tau_{f}}$ is the correct transmission probability and BER and $R$ are the bit error rate and transmission rate, respectively. $\mathrm{E}\left\{\tau_{\mathrm{n}-\mathrm{cci}}\right\}$ is defined similarly, with $\mathrm{E}\left\{\tau_{\text {sft }}^{\mathrm{sa}}\right\}$ replaced by $\mathrm{E}\left\{\tau_{\text {sft }}\right\}$.

In Fig. 3, the SFT durations with and without CCI are presented, for both AWGN and flat fading channels. As the $\frac{\tau_{f}}{\mathrm{E}\left\{\tau_{s}\right\}}$ ratio increases, the CCI case becomes highly advantageous. This advantage becomes significant when $\tau_{f}>5 \cdot \mathrm{E}\left\{\tau_{s}\right\}$, following an exponential trend. Under fading, the reduction in delay by $\mathrm{CCI}$ is higher than the reduction in delay by CCI under AWGN only; making CCI even more useful under fading. However, the gains in SFT duration vanish under low PU arrival rate or unconventionally low $\frac{\tau_{f}}{\mathrm{E}\left\{\tau_{s}\right\}}$ ratio.

Fig. 4 illustrates the effect of SU node density on SFT duration. SFT performance is investigated for two $\alpha$ values from Section VII-A, namely, 4 and 1, for the SU node density 


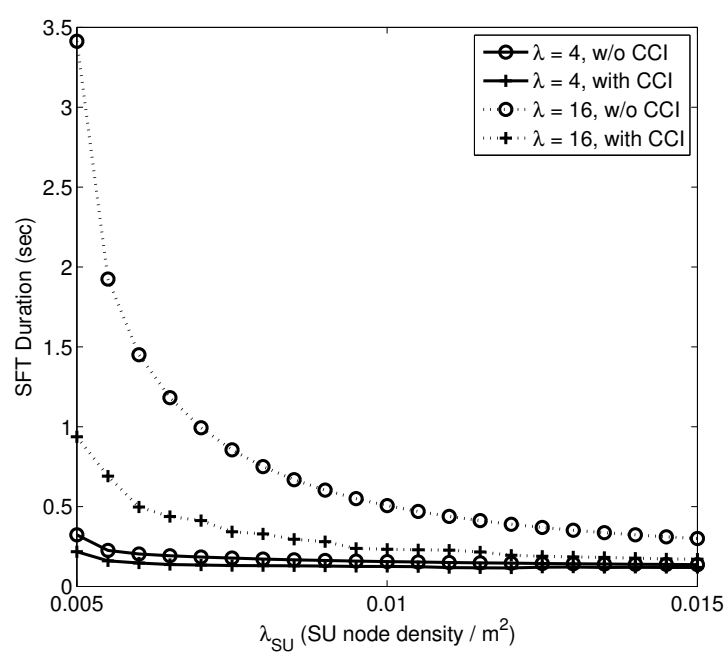

Fig. 4. SFT duration w.r.t. $\lambda_{\mathrm{SU}}$, with and w/o CCI. $E_{b} / N_{0}=10 d B$ at $\lambda_{\mathrm{SU}}=0.005, \lambda_{\mathrm{PU}}$ is assumed $\lambda_{\mathrm{PU}}=0.0125$

$\lambda_{\mathrm{SU}}=0.01$; resulting in total PU traffic values of 16 and 4 at that SU density. The SNR and total PU packet arrival rates for other $\lambda_{S U}$ values are calculated according to their $R_{c}$ values. In Fig. 4, increasing the $\lambda_{S U}$ decreases the SFT duration, since SU nodes become closer and links bear less PU traffic. CCI provides same SFT duration as the non-CCI case, at a lower SU density. This result is important for network design choices, for instance moderate node density and CCI incorporation could perform equally to high the node density.

Fig. 5 is a further investigation of the economical aspects of CCI incorporation. Inherently, the CCI has a hardware cost. Besides, an additional cost for the network-wide CCI channel allocation and maintenance can also be incurred, making the CCI cost hard to estimate. To this end, we investigate the total equivalent node cost per meter-square to achieve a given SFT duration, for varying CCI costs in terms of the regular full node cost. The non-CCI case becomes less and less costly and can become advantageous for certain CCI cost values, as the SFT duration constraint is relaxed. Logically, on the low SU density side, to meet a not very strict SFT requirement increasing node density should be the choice, since it has higher incremental utility in delay gains by enhancing the received power (and consequently BER) of transmission. For stringent SFT requirements one needs high SU density, but incremental utility of increasing node density on communication performance is monotonically decreasing. In that case, one needs CCI to obtain significant gains in delay. The conclusion is that, to achieve stringent SFT requirements, $\mathrm{CCI}$ is the key to a reduced cost, whereas with less stringent SFT constraints, solely increasing node density is less costly.

\section{Energy Efficiency}

Energy efficiency depends on $\tau_{s}, \tau_{f}$, and power consumption levels in these periods. We use two different spectrum sensing $\Upsilon_{s}$ and frame transmission $\Upsilon_{f}$ power level pairs, i.e., $\left\{\Upsilon_{s_{1}}=180 \mathrm{~mW}, \Upsilon_{f_{1}}=320 \mathrm{~mW}\right\}$, and $\left\{\Upsilon_{s_{2}}=\right.$

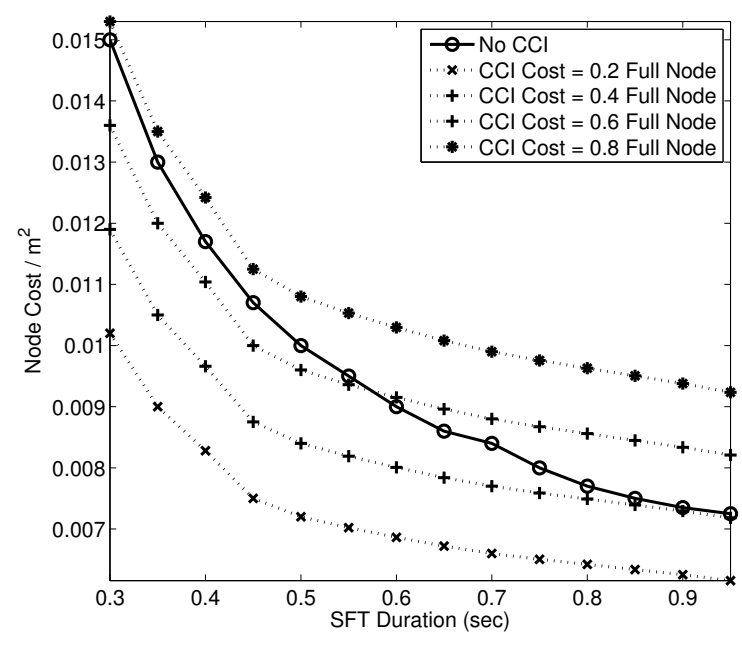

Fig. 5. Equivalent node cost, for a given SFT duration to be satisfied, for various CCI cost to full node cost ratios.

$\left.240 \mathrm{~mW}, \Upsilon_{f_{2}}=200 \mathrm{~mW}\right\}$, two CCI power consumption levels $\left(\Upsilon_{c c i_{1}}=100 \mathrm{~mW}\right.$ and $\Upsilon_{c c i_{2}}=300 \mathrm{~mW}$ ), and assume fixed $\mathrm{E}\left\{\tau_{s}\right\}$ of $5 \mathrm{~ms}$. The effects of CCI on energy efficiency are nontrivial since it consumes energy to decrease SFT duration; consequently saving energy to some extent. In Fig. 6(a) the energy efficiency for low traffic scenario $(\lambda=2)$ is considered. In cases where CCI consumes more power than normal transmission, energy efficiency is almost always degraded by CCI; meaning that under low traffic, gains of CCI cannot compensate its power consumption. However a CCI manageable by low power either outperforms or performs equally in terms of energy efficiency as its non-CCI counterparts. Fig. 6(b) considers the high traffic $(\lambda=8)$ scenario, in which low CCI power consumption cases usually outperform the regular non-CCI setups and high power CCI cases either underperform or perform similarly to regular non-CCI setups.

\section{Spectrum Efficiency}

The goodput performance of CRN with and without $\mathrm{CCI}$ is assessed in Fig. 6(c). We consider the goodput per energy consumed for an SFT as the spectrum efficiency metric. This ratio provides more meaningful results than bare goodput, assessing whether CCI brings in extra goodput in an energy efficient manner. Considered scenario comprises two PU traffic cases and two CCI power consumption levels. In the lower traffic case, depicted by dashed lines, CCI inclusion with power consumption equal to that of regular transmission results in lower goodput per energy, whereas CCI with low power consumption and non-CCI cases perform almost identically. Under high traffic, however, the CCI that requires lower power consumption than regular transmission brings in energy efficient goodput, whereas high power demanding CCI and non-CCI cases perform similarly. It is revealed that if the $\mathrm{CCI}$ energy consumption per node, including $\mathrm{CCI}$ radio consumption and common control channel maintenance cost across the network or local area, cannot be kept at a lower level 


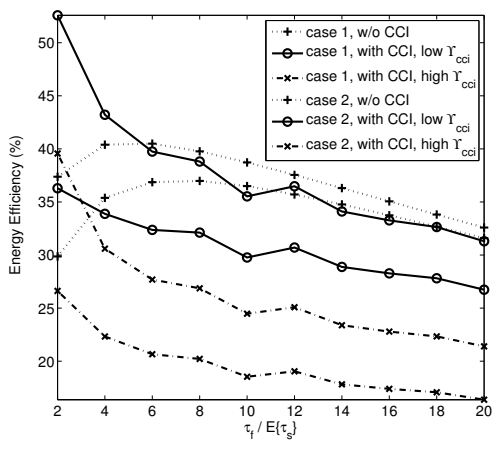

(a)

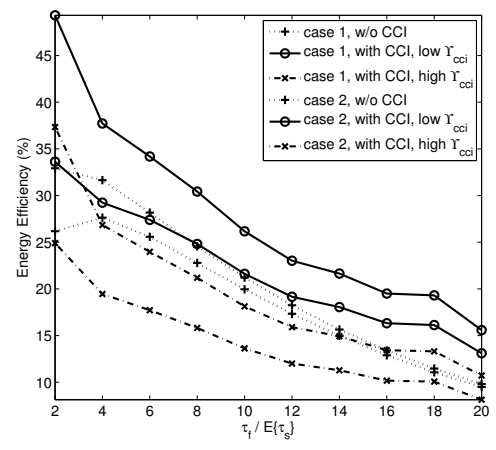

(b)

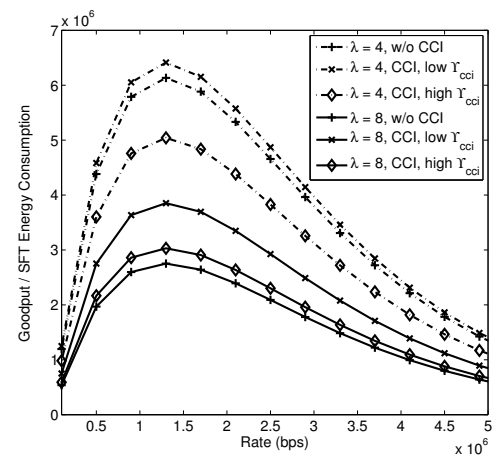

(c)

Fig. 6. Energy efficiency with and w/o CCI, for two transmission and sensing power level pairs under low (a) and high (b) PU traffic, Goodput/(SFT energy consumption) ratio (in bits/(second-Joule)), under different PU traffic and CCI power levels (c).

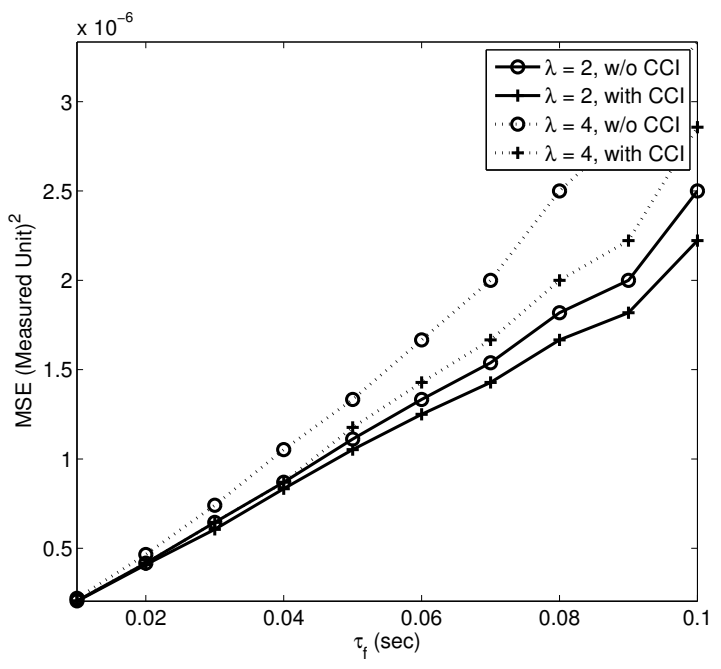

Fig. 7. Distributed sensing distortion performance with and without CCI, under different PU arrival rates.

than regular transmission consumption, then goodput gain of CCI comes at the cost of increased energy consumption.

\section{E. Distributed Event Sensing Performance}

Distributed event sensing distortion results are presented in Fig. 7. Sensing noise $\sigma_{k}^{2}$ is taken to be 0.01 for all sensors. Estimation interval $\tau_{e}$ is taken to be $1 \mathrm{sec}$. Number of samples per frame $S_{p}$ is set to 10 . Achieved mean square error (MSE) with CCI is expectedly lower than the one achieved without CCI. Nevertheless, the MSE gains by CCI heavily depend on $\frac{\tau_{f}}{\mathrm{E}\left\{\tau_{s}\right\}}$ ratio and PU traffic conditions. Since sensor nodes have lower ranges, we consider a lower PU traffic imposed upon them, than their CRN counterparts. Gains in MSE with CCI are not that bright, when $\tau_{f}$ is not an order greater than $\tau_{s}$. Gain in achieved MSE with CCI increase with increasing PU traffic, however not as drastically as their CRN equivalents.

\section{CONCLUSIONS}

In this paper, we analyze the effects of adding a dedicated common control interface for spectrum handoff in CRN. First, we build an analytical framework to characterize the time elapsed until a frame is successfully transmitted, namely, SFT duration, with and without CCI. The framework is extended to cover the effects of CCI on energy and spectrum efficiency, and distributed sensing performance. Implications of employment of CCI are presented for different network and CR conditions via numerical results. Following guidelines were revealed for CCI incorporation for spectrum handoff:

1) Significant gains in delay can be obtained with CCI, especially when $\frac{\tau_{f}}{\mathrm{E}\left\{\tau_{s}\right\}} \geq 10$.

2) In sparse ad hoc networks, the delay is dominated by BER, hence adding nodes will often be more cost effective than incorporating CCI. However, under moderate densities, incorporating CCI provides drastic delay gains, compared to further increasing node density.

3) The gains in goodput by CCI is not energy efficient unless there is high PU traffic and CCI can be managed at a lower power than the main radio.

4) $\mathrm{CCI}$ inclusion in $\mathrm{CRSN}$ is not wise, given low improvement in MSE and that CCI increases energy consumption, unless it operates under heavy PU traffic; which is seldom the case for sensor nodes with small ranges.

The provided framework can be used to perform detailed analysis of spectrum handoff efficiency with CCI utilization in terms of delay, energy, throughput, and estimation distortion for application specific network setups.

\section{REFERENCES}

[1] S. Haykin, "Cognitive Radio: Brain-empowered Wireless Communications," IEEE Journal on Selected Areas in Communications (JSAC), vol. 23, no. 2, pp. 201-220, February 2005.

[2] I. F. Akyildiz, W. Y. Lee, M. C. Vuran, and S. Mohanty, "NeXt Generation/Dynamic Spectrum Access/Cognitive Radio Wireless Networks: A Survey," Elsevier Computer Networks Journal, vol. 50, no. 13, pp. 2127-2159, January 2006.

[3] B. F. Lo, "A survey of common control channel design in cognitive radio networks," Elsevier Physical Communication, vol. 1, no. 4, pp. 26-39, 2011.

[4] K. R. Chowdhury, and I. F. Akyildiz, "OFDM-based Common Control Channel Design for Cognitive Radio Ad Hoc Networks," IEEE Transactions on Mobile Computing, vol. 10, no. 2, pp. 228-238, February 2011.

[5] B.F. Lo, I.F. Akyildiz, and A.M. Al-Dhelaan, "Efficient Recovery Control Channel Design in Cognitive Radio Ad Hoc Networks," IEEE Transactions on Vehicular Technology, vol. 59, no. 9, pp. 4513-4526, November 2010 
[6] N. Baldo, A. Asterjadhi, and M. Zorzi, "Dynamic Spectrum Access Using a Network Coded Cognitive Control Channel," IEEE Trans. on Wireless Communications, vol. 9, no. 8, pp. 2575-2587, 2010.

[7] L. Le, and E. Hossain, "OSA-MAC: A MAC Protocol for Opportunistic spectrum access in cognitive radio networks," in Proc. IEEE WCNC 2008, pp. 14261430, April 2008.

[8] Q. Zhao, L. Tong, A. Swami, and Y. Chen, "Decentralized Cognitive MAC for Opportunistic Spectrum Access in Ad Hoc Networks: A POMDP Framework, IEEE Journal on Selected Areas in Communications, vol. 25, no. 3, pp. 589-600, April 2007.

[9] Y. R. Kondareddy, and P. Agrawal, "Synchronized MAC Protocol For Multi-hop Cognitive Radio Networks," in Proc. IEEE ICC 2008, June 2008.

[10] H. Su, X. Zhang, "Cross-layer Based Opportunistic MAC Protocols for QoS Provisionings Over Cognitive Radio Wireless Networks," IEEE Journal on Selected Areas in Communications, vol. 26, no. 1, pp. 118$129,2008$.

[11] C. Wang, L. Wang, and F. Adachi, "Modeling and Analysis for Reactivedecision Spectrum Handoff in Cognitive Radio Networks," in Proc. IEEE Globecom 2010, December 2010.

[12] Y. Song, and J. Xie, "Performance Analysis of Spectrum Handoff for Cognitive Radio Ad Hoc Networks without Common Control Channel under Homogeneous Primary Traffic," in Proc. IEEE INFOCOM 2011, April 2011.

[13] L. Wang, C. Wang, and C. Chang, "Modeling and Analysis for Spectrum Handoffs in Cognitive Radio Networks," IEEE Transactions on Mobile Computing, to appear in 2012.

[14] V. K. Tumuluru, P. Wang, D. Niyato, and W. Song, "Performance Analysis of Cognitive Radio Spectrum Access with Prioritized Traffic," IEEE Transactions on Vehicular Technology, vol. 61, no. 4, pp. 18951906, May 2012.

[15] L. Wang, C. Wang, and K. Feng, "A Queueing-theoretical Framework for QoS-enhanced Spectrum Management in Cognitive Radio Network," IEEE Wireless Communications, vol. 18, no. 6, pp. 18-26, December 2011

[16] Y. Zhang, "Spectrum Handoff in Cognitive Radio Networks: Opportunistic and Negotiated Situations," in Proc. IEEE ICC 2009, June 2009.

[17] D. Willkomm, J. Gross, A. Wolisz, "Reliable Link Maintenance in Cognitive Radio Systems," in First IEEE International Symposium on New Frontiers in Dynamic Spectrum Access Networks, pp.371-378, 8-11 Nov. 2005.

[18] Suk-Un, E. Ekici, "Voluntary Spectrum Handoff: A Novel Approach to Spectrum Management in CRNs," in IEEE International Conference on Communications (ICC), pp.1-5, 23-27 May 2010.

[19] I.F. Akyildiz, B. F. Lo, and R. Balakrishnan, "Cooperative Spectrum Sensing in Cognitive Radio Networks: A Survey," Elsevier Physical Communication, vol. 4, no. 1, pp. 40-62, March 2011.

[20] K. Sriram and W. Whitt, "Characterizing superposition arrival processes in packet multiplexer for voice and data," IEEE Journal of Selected Areas in Communications, vol. 4, no. 6, pp. 833-846, Sep. 1986.

[21] Y. Chen, Q. Zhao, and A. Swami, "Joint Design and Separation Principle for Opportunistic Spectrum Access," in Proc. IEEE Asilomar Conference on Signals, Systems and Computers 2006, Oct. 2006.

[22] C. Chou, S. Shankar, H. Kim, and K. G. Shin, "What and How much to Gain by Spectrum Agility?", IEEE Journal of Selected Areas in Communications, vol. 25, no. 3, pp. 576-588, Apr. 2007.

[23] W. Y. Lee, and I. F. Akyildiz, "Optimal Spectrum Sensing Framework for Cognitive Radio Networks," IEEE Transactions on Wireless Communications, vol. 7, no. 10, pp. 3845-3857, 2008.

[24] D. Logothetis, K. S. Trivedi, and A. Puliafito, "Markov regenerative models," in Proc. Int. Computer Performance and Dependability Symp., Erlangen, Germany, pp. 134-143, 1995.

[25] O. B. Akan, O. B. Karli, and O. Ergul, "Cognitive Radio Sensor Networks," IEEE Network, vol. 23, no.4, pp. 34-40, July-August 2009.

[26] A. O. Bicen, V. C. Gungor, and O. B. Akan, "Spectrum-aware and Cognitive Sensor Networks for Smart Grid Applications," IEEE Communications Magazine, vol. 50, no. 5, pp. 158-165, May 2012.

[27] Z. Liang, S. Feng, D. Zhao, and X. Shen, "Delay Performance Analysis for Supporting Real-Time Traffic in a Cognitive Radio Sensor Network," IEEE Transactions on Wireless Communications, vol. 10, no. 1, pp. 325335, January 2011.

[28] M. C. Oto, and O. B. Akan, "Energy-efficient Packet Size Optimization for Cognitive Radio Networks," IEEE Transactions on Wireless Communications, vol. 11, no. 4, pp. 1544-1553, April 2012.

[29] J. A. Han, W. S. Jeon, and D. G. Jeong, "Energy-Efficient Channel Management Scheme for Cognitive Radio Sensor Networks," IEEE
Transactions on Vehicular Technology, vol. 60, no. 4, pp. 1905-1910, May 2011.

[30] A. O. Bicen, and O. B. Akan, "Reliability and Congestion Control in Cognitive Radio Sensor Networks," Elsevier Ad Hoc Networks, vol. 9, no. 7, pp. 1154-1164, Sept. 2011.

[31] A. O. Bicen, V. C. Gungor, and O. B. Akan, "Delay-sensitive and Multimedia Communication in Cognitive Radio Sensor Networks," Elsevier Ad Hoc Networks, vol. 10, no. 5, pp. 816-830, July 2012.

[32] J. M. Mendel, Lessons in Estimation Theory for Signal Processing, Communications, and Control. Englewood Cliffs, NJ: Prentice-Hall, 1995.

[33] M. Haenggi, "On distances in uniformly random networks," IEEE Trans. on Information Theory, vol. 51, no. 10, pp. 3584-3586, Oct. 2005.

[34] A. Goldsmith, Wireless Communications. Cambridge, U.K.: Cambridge Univ. Press, 2004.

[35] M. K. Simon, M. S. Alouini, Digital Communication over Fading Channels A Unified Approach to Performance Analysis. Hoboken, NJ: 1st ed., Wiley, 2000.

[36] Ying-Chang Liang; Yonghong Zeng; Peh, E.; Anh Tuan Hoang; , "Sensing-Throughput Tradeoff for Cognitive Radio Networks," in Proc. IEEE ICC '07 International Conference on Communications, pp.53305335, 24-28 June 2007.

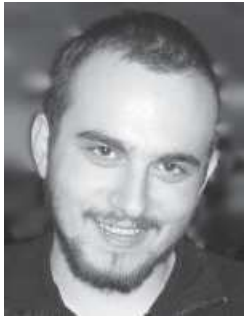

A. Ozan Bicen (S'08) received the B.Sc. and M.Sc degrees in Electrical and Electronics Engineering from Middle East Technical University, Ankara, Turkey, in 2010 and from Koc University, Istanbul, Turkey in 2012, respectively. Currently, he is a Ph.D. candidate in the Broadband Wireless Networking Laboratory, School of Electrical and Computer Engineering, Georgia Institute of Technology. His current research interests include design, and analysis of molecular communication systems and networks.

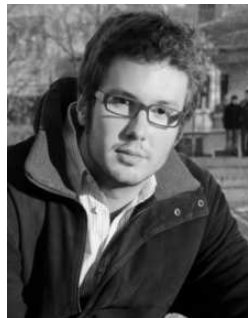

Ecehan B. Pehlivanoglu (S'12) received the B.Sc. degree in Electrical and Electronics Engineering from Middle East Technical University, Ankara, Turkey, in 2011. Currently he is a research assistant at Next-Generation Wireless Communication Laboratory (NWCL) and pursuing his Ph.D. degree at the Electrical and Electronics Engineering Department, Koc University, Istanbul. His research interests include cognitive radio, cognitive radio sensor networks and nanoscale communications.

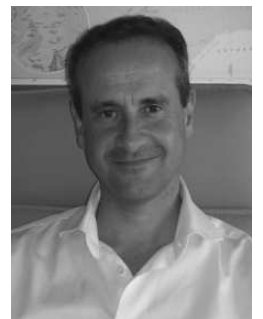

Sebastià Galmés (M'10) received electrical engineers degree from the Polytechnic University of Catalonia (Barcelona, Spain) in 1989, and the Ph.D. degree in computer science from the University of the Balearic Islands (Palma, Spain) in 1999. He is currently Associate Professor in the Department of Mathematics and Computer Science at the University of the Balearic Islands. His current research interests focus on wireless sensor networks, molecular communications and nano-networks, cognitive radios and wireless networks in general.

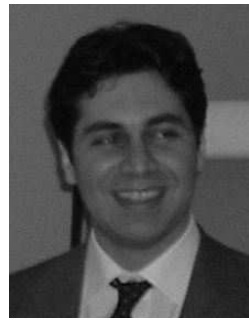

Ozgur B. Akan (M'00-SM'07) received the Ph.D. in electrical and computer engineering from Georgia Institute of Technology, Atlanta, in 2004. He is currently a Full Professor with the Department of Electrical and Electronics Engineering and the Director of the Next-generation and Wireless Communications Laboratory, Koc University, Istanbul, Turkey. His current research interests include wireless communications, nanoscale and molecular communications, and information theory. 